Bundesgesundheitsbl 2021 · 64:1328-1329 https://doi.org/10.1007/s00103-021-03411-z (c) Springer-Verlag GmbH Deutschland, ein Teil von Springer Nature 2021

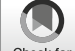

Bekanntmachung des Umweltbundesamtes

\title{
Ergänzung der Empfehlung "Umgang mit per- und polyfluorierten Alkylsubstanzen (PFAS) im Trinkwasser" vom 26. August 2020
}

\section{Empfehlung des Umweltbundesamtes nach Anhörung der Trinkwasserkommission}

im Trinkwasser notwendig, wenn man sich an der für Lebensmittel veröffentlichten EFSA-Stellungnahme orientiert.

Erstmalig sind bereits in der TW-RL europaweit verbindliche Parameterwerte für PFAS im Trinkwasser festgelegt. Die TW-RL sieht für PFAS zwei neue Parameter vor: einen Summenwert für 20 definierte Einzelverbindungen von PFAS $\left(P F A S_{\Sigma 20}\right)$ sowie einen Summenwert für PFAS als gesamte Substanzklasse $\left(\right.$ PFAS $\left._{\text {gecant }}\right)$.

- $P F A S_{\Sigma 20}=0,10 \mu \mathrm{g} / \mathrm{l}$

- $P F A S_{\text {gesamt }}=0,50 \mu \mathrm{g} / \mathrm{l}$

\section{Weitere Schritte zur Vorbereitung einer Regulierung von PFAS im Trinkwasser}

\section{PFAS in der EFSA-}

Veröffentlichung und der EUTrinkwasserrichtlinie

In ihrem im September 2020 veröffentlichten Gutachten zu PFAS leitet die EFSA eine aktualisierte tolerierbare wöchentliche Aufnahmemenge (TWI) für die Summe von vier PFAS $\left(P F A S_{\Sigma 4}\right)$, nämlich Perfluoroktansäure (PFOA), Perfluornonansäure (PFNA), Perfluorhexansulfonsäure $(\mathrm{PFHxS})$ und Perfluoroktansulfonsäure (PFOS), von 4,4 Nanogramm (ng) pro Kilogramm $(\mathrm{kg})$ Körpergewicht pro Woche ab [2]. Der TWI bezieht sich auf die Summe aller Aufnahmepfade und macht u.a. eine Anpassung der Grenzwerte für PFAS
Die verschiedenen Aktivitäten zur Verbesserung des Erkenntnisstandes hinsichtlich PFAS werden durch das Umweltbundesamt zusammengeführt, damit diese im Rahmen der Umsetzung der TW-RL in einer Novelle der Trinkwasserverordnung angemessen berücksichtigt werden können.

- Gegenwärtig werden geeignete Untersuchungsverfahren zur Bestimmung des Parameters PFAS $_{520}$ im Rahmen eines Normungsverfahrens auf EUEbene (CEN) bearbeitet, wobei bestimmte Anforderungen (z.B. Bestimmungs- und Nachweisgrenzen) konkretisiert werden sollen. Schon jetzt lassen sich die 20 Einzelsubstanzen aus der Gruppe der $P F A S_{\Sigma 20}$ weitgehend mit sog. Haus-Verfahren auf Basis der Deutschen Norm DIN 38407-42 analysieren, jedoch ist eine wünschenswerte Bestimmungsgrenze von $0,001 \mu \mathrm{g} / \mathrm{l}$ (Mikrogramm pro Liter) je Einzelverbindung in der Praxis derzeit noch schwer zu erreichen.

- Gegenstand aktueller Forschung ist es, die Betroffenheit der Trinkwasserversorgung in Deutschland hinsichtlich des Vorkommens von PFAS im Rohwasser und ihrer Entfernbarkeit durch technische Aufbereitungsverfahren genauer einzuschätzen.

- Die fachliche Meinungsbildung hinsichtlich der Festsetzung von Grenzwerten wird intensiv fortgesetzt. Dazu gehören Fragen wie eine evtl. Gewichtung für kurz- und langkettige PFAS für den Parameterwert $P F A S_{\Sigma 20}$ unter Berücksichtigung ihrer unterschiedlichen Toxizität.

- Den Ländern und anderen Beteiligten wird empfohlen, für den Parameter $P F A S_{\Sigma 20}$ analytische Kapazitäten zu etablieren und sich im Rahmen eines Roh- und Trinkwasser-Monitorings einen Überblick zum Vorkommen zu verschaffen, damit im Hinblick auf die Novelle der deutschen Trinkwasserverordnung mögliche Regelungsbedarfe erkannt und frühzeitig 
Maßnahmen ergriffen werden können. Hiermit sollten auch flächendeckend Eintragsquellen von PFAS in die Trinkwasserressourcen (wie bspw. Flughäfen, Militäreinrichtungen, Klärschlammausbringung, Industrieanlagen) identifiziert werden (s.a. [1]).

Das Umweltbundesamt wird über den Fortgang der Aktivitäten weiter informieren.

\section{Weiterentwicklung der vorläufigen nationalen Bewertung}

Die vorübergehenden Maßnahmenwerte für PFOS und PFOA [4] wurden vom Umweltbundesamt bereits Ende 2019 in Erwartung der Neubewertung durch die EFSA festgelegt und tragen dem Vorsorgegedanken Rechnung. Eine Weiterentwicklung der bestehenden vorläufigen Bewertung des Umweltbundesamts, bei welcher Leitwerte und Gesundheitliche Orientierungswerte (GOW) für verschiedene Verbindungen festgelegt wurden [5], kann sich infolge der Festlegung eines Grenzwerts für PFAS in der Trinkwasserverordnung ergeben. Wie in der Empfehlung vom 26. August 2020 dargestellt [1], gelten Leitwerte, GOW sowie die Maßnahmenwerte für PFOS und PFOA daher zunächst weiter.

Bei der Aktualisierung der Bewertung von PFAS-Einzelsubstanzen wird der von der EFSA empfohlene Wert für die tolerierbare wöchentliche Aufnahmemenge für bestimmte PFAS angemessen $\mathrm{zu}$ berücksichtigen sein. Dies könnte mindestens für die vier hierbei betrachteten PFAS-Einzelverbindungen PFOA, PFNA, PFHxS und PFOS zu Empfehlungen für Höchstwerte oder Grenzwerte führen, die sehr deutlich unter den bisherigen Leitwerten, vorübergehenden Maßnahmenwerten bzw. allgemeinen Vorsorgewerten liegen würden.

Bei der Beurteilung von PFAS im Trinkwasser unter Berücksichtigung des TWI-Wertes der EFSA sind neben dem Ansatz des Minimierungsgebotes gemäß $\$ 6$ Absatz 3 der Trinkwasserverordnung auch die Umsetzbarkeit und die Verhält- nismäßigkeit von Maßnahmen zu berücksichtigen.

\section{Anhang}

\section{Relevante Fristen zur Umsetzung der EU-Trinkwasserrichtlinie in nationales Recht}

Die Mitgliedstaaten müssen die Bestimmungen der TW-RL bis spätestens zum 12. Januar 2023 in nationales Recht umsetzen. Der Bundesgesetzgeber wird dafür unter anderem die Trinkwasserverordnung novellieren. Im Zusammenhang mit den Parameterwerten zu PFAS sind dabei folgende weitere Fristen von Bedeutung:

- Bis zum 12. Januar 2024 legt die EUKommission gemäß Artikel 13 Absatz 7 der TW-RL technische Leitlinien bezüglich der Analysenverfahren zur Überwachung der PFAS fest.

- Der Parameterwert für PFAS gesamt $_{\text {gilt }}$ gemäß den Anmerkungen in Anhang 1 Teil B erst, wenn die technischen Leitlinien bezüglich der Analysenverfahren zu ihrer Überwachung zur Verfügung stehen. Die Mitgliedstaaten können anschließend entscheiden, einen der Parameter $P_{\text {PFAS }}$ gesamt $_{\text {oder }}$ PFAS $S_{\Sigma 20}$ oder beide zu verwenden.

- Bis zum 12. Januar 2026 haben die Mitgliedsstaaten die nötigen Maßnahmen nach Artikel 25 Absatz 1 der TW-RL zu ergreifen, um sicherzustellen, dass Trinkwasser den festgelegten Parameterwerten für die PFAS fristgerecht entspricht.

- Ab dem 13. Januar 2026 sind die Wasserversorger nach Artikel 25 Absatz 2 der TW-RL verpflichtet, PFAS in ihren Überwachungsprogrammen für Trinkwasser gemäß Artikel 13 der TW-RL zu berücksichtigen.

- Bis zum 12. Juli 2027 sind die Risikobewertung und das Risikomanagement der Einzugsgebiete von Entnahmestellen nach Artikel 7 Absatz 4 der TW-RL das erste Mal durchzuführen. Aus Artikel 8 Absatz 4 der TW-RL folgt, dass auch die dort genannten Maßnahmen (unter anderem Präventivmaßnahmen/Minderungsmaßnahmen/Überwachungsmaßnahmen/Festlegung und Erweiterung von Schutzgebieten) ergriffen werden müssen. Auf Basis von Artikel 7 Absatz 7 der TW-RL sind Mitgliedstaaten nicht daran gehindert, dass nach der Erkennung und Bewertung von Risiken die erforderlichen Maßnahmen möglichst bald, also auch früher, ergriffen werden.

\section{Literatur}

1. Umweltbundesamt Empfehlung des Umweltbundesamtes - Umgang mit per- und polyfluorierten Alkylsubstanzen (PFAS) im Trinkwasser (2020). https://www.umweltbundesamt.de/sites/default/ files/medien/5620/dokumente/twk_200826_ empfehlung_pfas_final.pdf;. Zugegriffen: 10. Juni 2021

2. EFSA (2020) EFSA: Risk to human health related to the presence of perfluoroalkyl substances in food. Efsa Journal Eur Food Saf Auth 18(9):e6223. https://doi.org/10.2903/j.efsa.2020.6223

3. EU-Richtlinie: Richtlinie (EU) 2020/2184 des Europäischen Parlaments und des Rates. vom 16. Dezember 2020 über die Qualität von Wasser für den menschlichen Gebrauch (Neufassung) (2020)

4. Umweltbundesamt Stellungnahme zu einem vorübergehenden Maßnahmenwert für PFOA und PFOS (2019). https://www.umweltbundesamt. de/sites/default/files/medien/421/dokumente/ stellungnahme_pfoa_pfos_0.pdf;. Zugegriffen: 10 . Juni 2021

5. Umweltbundesamt (2017) Fortschreibung der vorläufigen Bewertung von per- und polyfluorierten Chemikalien (PFC) im Trinkwasser: Empfehlung des Umweltbundesamtes nach Anhörung der Trinkwasserkommission. Bundesgesundheitsblatt Gesundheitsforschung Gesundheitsschutz 60(3):350-352. https://doi.org/10.1007/s00103016-2508-3 